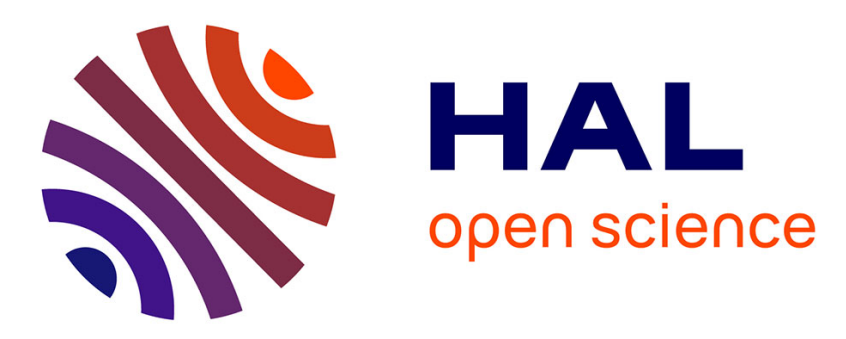

\title{
Guard Time Optimization for Capacity Maximization of BPSK Impulse UWB Communications
}

\author{
Abdallah Hamini, Jean-Yves Baudais, Andrea M. Tonello, Jean-François \\ Hélard
}

\section{- To cite this version:}

Abdallah Hamini, Jean-Yves Baudais, Andrea M. Tonello, Jean-François Hélard. Guard Time Optimization for Capacity Maximization of BPSK Impulse UWB Communications. Journal of Communication, 2014, 9 (2), pp.188-197. 10.12720/jcm.9.2.188-197 . hal-01065658

\section{HAL Id: hal-01065658 \\ https://hal.science/hal-01065658}

Submitted on 18 Sep 2014

HAL is a multi-disciplinary open access archive for the deposit and dissemination of scientific research documents, whether they are published or not. The documents may come from teaching and research institutions in France or abroad, or from public or private research centers.
L'archive ouverte pluridisciplinaire HAL, est destinée au dépôt et à la diffusion de documents scientifiques de niveau recherche, publiés ou non, émanant des établissements d'enseignement et de recherche français ou étrangers, des laboratoires publics ou privés. 


\title{
Guard Time Optimization for Capacity Maximization of BPSK Impulse UWB Communications
}

\author{
Abdallah Hamini ${ }^{2,3}$, Jean Yves Baudais ${ }^{1}$, Andrea M. Tonello², and Jean-François Hélard ${ }^{3}$ \\ ${ }^{1}$ National Center for Scientific Research (CNRS), IETR, UMR 6164, F-35708 Rennes, France \\ ${ }^{2}$ DIEGM - University of Udine viadelle Scienze, 20833100 - Udine - Italy \\ ${ }^{3}$ Université européenne de Bretagne, INSA, IETR, UMR 6164, F-35708 Rennes, France \\ Email: abdallah.hamini@insa-rennes.fr, jean-yves.baudais@insa-rennes.fr, tonello@uniud.it, \\ jean-francois.helard@insa-rennes.fr.
}

\begin{abstract}
Usually, for the design of UWB systems, the symbol duration is chosen larger than the delay of the channel impulse response, in order to avoid the ISI (inter symbol interference). However, this approach does not maximize the system capacity. An adaptation of the guard time (GT) is a flexible mean of exploiting system resources efficiently in a multi-path fading environment. The optimal guard time length in BPSK impulse UWB communications is obtained by exhaustively searching for the guard time that maximizes capacity. This approach is complex since it has to be implemented for each channel realization. To reduce this complexity, in this paper we present new optimization methods. The first method assumes the fading channel to be partitioned into classes. Then, a given GT length for each channel class is used. The second optimization method provides a guard time for each channel realization. However, the GT is obtained by looking at simplified metrics which are based on the channel delay spread, the received signal energy, or on an approximation of the capacity formula. Simulation results are performed for UWB communications over WiMedia channels and they show that significant gains are achievable with the proposed guard time adaptation w.r.t. to the use of a constant guard time.
\end{abstract}

Index Terms - System capacity, pulse design, guard time, BPSK, UWB

\section{INTRODUCTION}

The demand for new services and applications in communication systems, as well as the number of users, are steadily increasing. This growth involves a great need of data rate increase offered by the communication system. The system capacity is an important parameter for the design and evaluation of wireless networks. Recently, the UWB (ultra wide band) technique has been widely studied for wireless communications [1], [2]. UWB communications offer very high data rates due to the use of a wide band and the robustness to multi-path fading [3]. The system should be designed so that capacity is maximized. The Gaussian monocycle was initially proposed and has been widely used in impulsive UWB systems [4], [5]. In typical impulsive UWB system design, the symbol duration is larger than the maximum

Manuscript received September 9, 2013; revised February 16, 2014. Corresponding author email: abdallah.hamini@insa-rennes.fr. doi: $10.12720 /$ jcm.9.2.188-197 channel impulse response duration so that the intersymbol interference can be neglected [6], [7]. To this end, a guard time is added after the pulse transmission in order to avoid ISI. The optimization of the guard time is not generally considered since typically the guard time is set at a duration longer than the maximum channel duration [8]. However, this approach is energy and capacity inefficient. In fact, to maximize the capacity, the system does not necessarily need a large guard time. That is, the system can tolerate an amount of interference in order toreduce the guard time so that the system capacity can be improved. An adaptation of the guard time is a flexiblemean of exploiting system resources efficiently especially in a varying multi-path fading environment. The case of a guard interval shorter than the channel impulse response has been considered in OFDM systems [9], [10]. Following a similar concept, in this paper we report an analysis of the guard time optimization in impulsive UWB transmission. The optimization problem pursues the maximization of capacity. Since the channel response varies with time and position, ideally the guard time should be adaptively chosen. However, this method is computationally intense. To reduce the complexity, two methods are proposed in this paper. The first method partitions the channel into classes. Each class collects channel responses that provide a certain average attenuation and delay spread. Essentially, each class is representative of a certain environment as it is done in the WiMedia channel model [11]. Then, a single guard time value for each channel class can be defined and used. The second method provides an adaptive guard time for all channel realizations. However, the guard time is obtained by looking at simplified metrics that are based on the channel delay spread, the received signal energy and on the use of an approximation of the capacity formula. With these metrics the computation of the guard time is simplified and the method can be applied to any channel model.

The reminder of this paper is as follows. The description of the communication model is presented in Section II. The typical channel model used in UWB is introduced in Section III. In Section IV, the capacity calculation and the guard time optimization are introduced. In addition, the statistical analysis of the 
optimal guard time is provided. In Section V, the first method for guard time optimization is presented. Section VI introduces an optimization method for the guard time design with low complexity. Some alternative metrics are proposed to adjust the guard time in order to reduce the system complexity. Section VII describes the simulation setup, numerical results and provides an analysis of the results. Finally, Section VIII concludes this paper.

\section{COMMUNiCATION MODEL}

We consider a single user system model with BPSK (binary phase shift keying) signalling so that the transmitted signal can be written as [12]

$$
x(t)=\sum_{k} b_{k} g\left(t-k T_{b}\right)
$$

where $b k= \pm 1$ denotes the information bit transmitted in the frame $k$ and $T_{b}$ is the bit period (frame duration). We incorporate the differential effects of the transmission, and receive antennas into $g(t) . g(t)$ is assumed to be the second derivative of the Gaussian pulse

$$
g(t)=\left(1-\pi\left(\frac{t-\frac{T_{p}}{2}}{T_{0}}\right)^{2}\right) \exp \left(-\frac{\pi}{2}\left(\frac{t-\frac{T_{p}}{2}}{T_{0}}\right)^{2}\right)
$$

where $T_{p}$ is the monocycle pulse duration, and $T_{0}$ is the width of the pulse. We further insert a guard time $T_{g}$ between pulses. The bit duration fulfills the relation $T_{b}=$ $\left(T_{p}+T_{g}\right)$. The inter-symbol interference is avoided when $T_{b} \geq T_{p}+\tau_{\max }$, where $\tau_{\max }$ is the maximum delay (duration) introduced by the channel. At the receiver side, we first deploy a bandpass front-end filter to suppress the out of band noise. Then, the received signal, in the single user case, can be written as

$$
z(t)=\sum_{k} b_{k}(g \star h)\left(t-k T_{b}\right)+\eta(t)
$$

where $(g \star h)(t)$ is the convolution of the waveform filter $g(t)$ by the impulse response of the channel $h(t)$. The additive noise $\eta(t)$ is assumed to be a stationary zero mean Gaussian process. Further, in the following, we consider it to be white in the useful signal band. Let us suppose that the received signal is passed first through a matched filter $e(t)$

$$
y(t)=(e \star z)(t)
$$

The optimum filter from the point of view of SNR maximization is the matched filter [13]. The matched filter is adapted to the pulse and to the channel response. It is obtained by correlating the transmit pulse and the channel response. We furthermore assume to use a noise whitening filter (included in the impulse response $e(t)$ ) so that the sequence of samples at the output can be written as

$$
y_{n}=y\left(n T_{b}\right)=\sum_{k} b_{n-k} a_{k}+w_{n}
$$

where $a k$ are the inter-symbol interference amplitude coefficients, while $w_{n}$ is the sequence of i.i.d. Gaussian noise samples with zero mean and variance $N_{0}$. ISI is generated when the guard time is shorter than the channel duration.

\section{Channel Model (WiMedia ChanNEL)}

We consider UWB channels with frequency selective fading [14]. In particular, we use the model adopted by the IEEE 802.15.3a committee for the evaluation of UWB physical layer proposals [11]. The model defines four classes each characterized by line-of-sight (LOS) or nonline-of-sight (NLOS), a certain mean excess delay, RMS delay spread and distance between the transmitter and the receiver, as summarized in Table. I.

TABLE I: UWB CHANNEL CHARACTERISTICS

\begin{tabular}{lcccc}
\hline \hline & CM1 & CM2 & CM3 & CM4 \\
\hline Mean excess delay(ns) & 5.05 & 10.38 & 14.18 & 25.1 \\
Delay spread(ns) & 5.28 & 8.03 & 14.28 & 25 \\
Distance(m) & $<4$ & $<4$ & $4-10$ & $4-10$ \\
LOS/NLOS & LOS & NLOS & NLOS & NLOS \\
\hline \hline
\end{tabular}

This model is a modified version of Saleh-Valenzuela model for indoor channels, fitting the properties of measured UWB channels. A log-normal distribution is used for the multi-path gain magnitude. In addition, independent fading is assumed for each cluster and each ray within the cluster. The impulse response of the multipath model is given by

$$
h(t)=G \sum_{z=0}^{Z} \sum_{p=0}^{P} \alpha(z, p) \delta(t-T(z)-\tau(z, p))
$$

where $G$ is the attenuation due to log-normal shadowing, $T$ is the delay of cluster $z, \alpha(z, p)$ and $\tau(z, p)$ represent the gain and the delay of the multi-path component $p$ of cluster $z$. The cluster and the path arrival times are modelled according to a Poisson arrival process. The path amplitude follows a log-normal distribution each with arrival rates and decay factors chosen to match different usage scenarios and to fit line-of-sight and non-line-ofsight cases. More details can be found in [11].

\section{GUARD TIME OPTIMIZATION}

\section{A. Capacity Calculation}

To evaluate the impact of the guard time length on the system performance, we define the optimum value of guard time that maximizes the system capacity. The capacity is defined as the maximum of the mutual information

$$
C=\max _{p(X)} I(X, Y)
$$

In the case of BPSK, the mutual information is maximized for equi-probable symbols. Let us now compute the mutual information as a function of the bit energy $E b$, the Gaussian noise variance $N_{0}$ and the inter- 
symbol interference that is generated when the guard time is shorter than the channel duration. The mutual information is

$$
I(X, Y)=S(Y)-S(Y \mid X)
$$

where both entropies are defined as [15]

$$
S(Y \mid X)=\frac{1}{2} \log _{2} 2 \pi e N_{0}
$$

and

$$
S(Y)=-E\left[\log _{2} p(Y)\right]
$$

where the probability $p(Y)$ is defined as

$$
\begin{aligned}
p(Y) & =\left(\frac{1}{2^{n+1}} \frac{1}{\sqrt{2 \pi}}\right. \\
& \left.\sum_{s=1}^{2} \sum_{i=1}^{2^{n}} \exp \left(-\frac{\left(y-b_{s} A+\sum_{j=1}^{n} a_{j} \alpha_{j, i}\right)^{2}}{2}\right)\right)
\end{aligned}
$$

where $b_{s}= \pm 1, n$ is the number of interfering bits (due to a short guard time), $a_{j}$ is the interference amplitude of bit $j$, $\alpha_{i, j}$ is the bit value equal to \pm 1 . Since there are $n$ binary interferers we have that $i=1 \ldots 2^{n}$. Furthermore, $A=$ $\sqrt{S N R}=\sqrt{E_{b} / N_{0}}$ Then, the capacity measured in bit/s/ $\mathrm{Hz}$ achieved in the case of BPSK is

$$
\begin{aligned}
C\left(T_{g}\right) & =-\frac{1}{T_{b} B}\left(E \left[\operatorname { l o g } _ { 2 } \left(\frac{1}{2^{n+1}} \frac{1}{\sqrt{2 \pi}}\right.\right.\right. \\
& \left.\left.\sum_{s=1}^{2} \sum_{i=1}^{2^{n}} \exp \left(-\frac{\left(y-b_{s} A+\sum_{j=1}^{n} a_{j} \alpha_{j, i}\right)^{2}}{2}\right)\right)\right] \\
& \left.-1 / 2 \log _{2}\left(2 \pi e N_{0}\right)\right)
\end{aligned}
$$

where $B$ is the channel bandwidth. The Monte Carlo integration is needed to compute $C\left(T_{g}\right)$. The detailed calculation of capacity is provided in Appendix A. It follows that the optimal guard time length can be computed as

$$
T_{g}^{*}=\arg \max _{T_{g}} C\left(T_{g}\right)
$$

The evaluation of the argument in (13) is computationally intense because it requires a Monte Carlo integration for the evaluation of the expectation for each single value of guard time. Therefore, it is important to derive a simplified solution with lower complexity.

\section{B. Capacity Optimization}

To begin our analysis, we consider in this section the capacity of the BPSK UWB system assuming a Gaussian pulse with duration $T_{p}=5 \mathrm{~ns}$. Ideal knowledge of the channel is assumed at the receiver side. Then, the capacity is evaluated assuming the model in Section III. In particular, a randomly picked impulse response within the class CM1 is considered to obtain the capacity in Fig.
1. The capacity has a function of the SNR and the guard time. As shown in Fig. 1, for a certain SNR the capacity depends on the guard time. The optimal guard time varies for each SNR value although this variation is more contained. In Fig. 2, we consider an SNR equal to $10 \mathrm{~dB}$ and a randomly picked channel impulse response per class. The capacity increases as the guard time increases up to a given value of $T_{g}^{*}$. The maximum capacity is achieved with the optimal guard time $T_{g}^{*}$. Interestingly, the optimal guard time is different for each channel realization. For the CM1 channel response, the optimal guard time value is $2 \mathrm{~ns}$. This value increases to $5 \mathrm{~ns}$ for the CM2 channel, to $8 \mathrm{~ns}$ for the CM3 channel and to 11 ns for the CM4 channel. The convex (although not strictly) behaviour of the capacity can be explained by observing that the capacity is dependent on both the guard time and the interference. When the guard time length increases there is a logarithmic increase of the capacity with the increase of the signal-to interferenceplus- noise ratio which is however counterbalanced by the linear decrease with the multiplicative factor $T_{b-1}$. Overall, the system capacity with the CM1 channel model is superior to the system capacity with other channel models. This channel has lower time dispersion so that a shorter guard time is required.

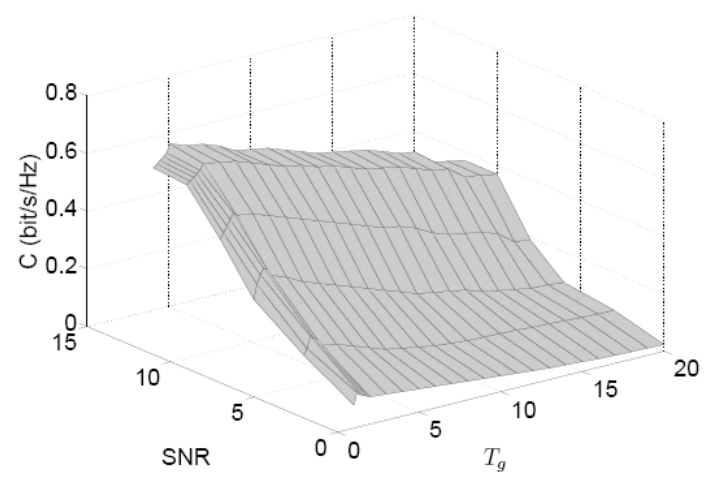

Fig. 1. Capacity vs SNR vs $T_{g}$ over the CM1 channel model.

We now turn our attention to the comparison between the conventional system that uses a guard time longer than the maximum channel excess delay $\tau_{\max }$ and the system with guard time optimally adapted. Assuming the same channel responses of Fig. 2, we report in Table. II the capacity achieved with the two design methods. With opti-mal adaptation of the guard time significant performance improvements are attainable. The gain factor is equal to 3.3 in the CM1 channel. This gain increases to 4.3 in the CM2 channel, to 5.8 in the CM3 channel and to 6.6 in the CM4 channel model. As explained, the optimal guard time depends on the channel realization. Fig. 3 shows the measured CDF (cumulative distribution function) of the optimal capacity according to (12) when the guard time is adaptively chosen for each channel realization. Fig. 4 presents the measured $\mathrm{CDF}$ of the optimal guard time according to (13). For the CM1 channel model, the guard time is always shorter than $6 \mathrm{~ns}$. 
This value increases to $8.5 \mathrm{~ns}$ for the CM2 channel model, to $14.5 \mathrm{~ns}$ for the CM3 channel model and to $20 \mathrm{~ns}$ for the CM4 channel model. In practice the adaptation requires that the receiver calculates the value of guard time for a certain channel realization and then it feeds back such an information to the transmitter. The procedure is applicable in slowly time variant channels but it is nevertheless complex. In order to simplify the approach, in the next sections we will describe other approaches.

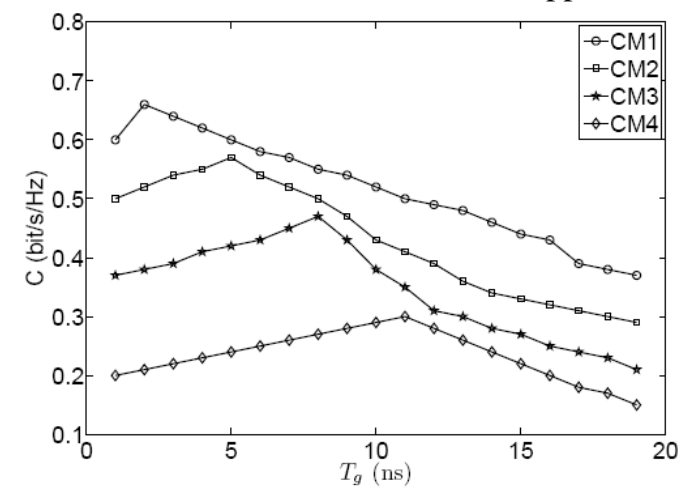

Fig. 2. Capacity vs guard time, $S N R=10 \mathrm{~dB}$.

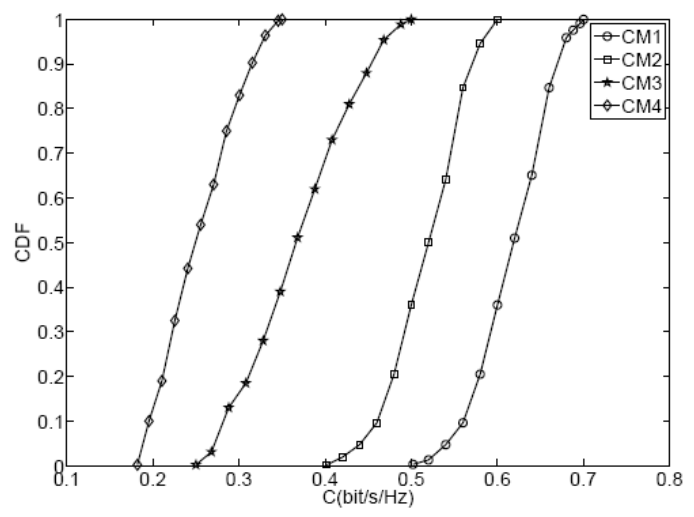

Fig. 3. CDF of system capacity, $\mathrm{SNR}=10 \mathrm{~dB}$.

TABLE II: CAPACITY COMPARISON

\begin{tabular}{ccccc}
\hline \hline & $\left(T_{g}^{*}\right) \mathrm{ns}$ & $C\left(T_{g}^{*}\right)$ & $\left(\tau_{\max }\right) \mathrm{ns}$ & $C\left(\tau_{\max }\right)$ \\
\hline CM1 & 2 & 0.66 & 35 & 0.2 \\
CM2 & 5 & 0.57 & 55 & 0.13 \\
CM3 & 8 & 0.47 & 75 & 0.08 \\
CM4 & 11 & 0.3 & 115 & 0.045 \\
\hline \hline
\end{tabular}

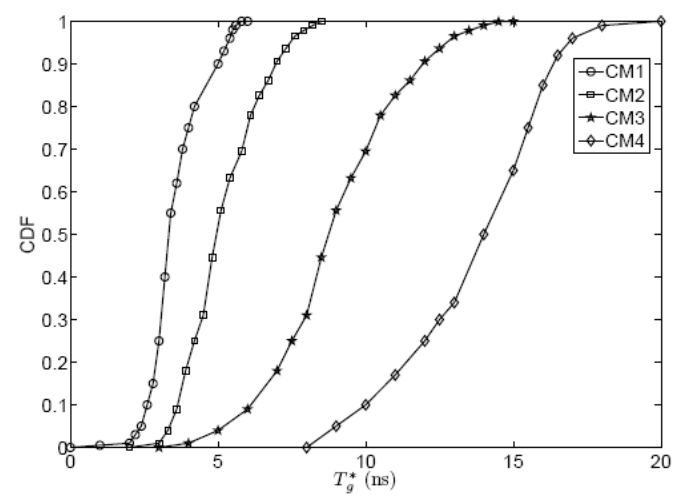

Fig. 4. $C D F$ of capacity optimal guard time, $S N R=10 \mathrm{~dB}$.

\section{REDUCED Number OF GuARD TIME VALUES}

In order to reduce complexity, we may want to use a small number of guard time lengths and adapt among these values. The idea is to precompute a single guard time length according to the channel class we are supposed to operate in. This is possible by choosing the guard time based on the evaluation of the CDF of the optimal guard time presented in Fig. 4. We propose that the optimal guard time duration does not vary significantly within the same class. Hence, we propose, for a given channel class, to choose a single value of guard time for all channel realizations. The specific guard time is chosen to be the value of $T_{g}^{*}$ for which the CDF of the optimal guard time is $99 \%$. We denote this value as $T_{g^{(99)} \text { i }}$ i.e., the 99th percentile of the guard time optimal $T_{g}^{*}$ Alternatively, we can consider the median value of the CDF of the optimal guard time. We denote it with $T_{g(50 \%)}$. It follows that in both cases, the guard time is selected depending on the class we are operating in. Although, for illustrative examples we consider the statistical WiMedia channel model, the design approach can be applied in other application scenarios for which a statistical channel model is available. To compare the performance, we define the relative capacity loss w.r.t. the optimal value, as follows

$$
\epsilon_{c}=\frac{C\left(T_{g}^{*}\right)-C\left(\bar{T}_{g}\right)}{C\left(T_{g}^{*}\right)}
$$

where $\bar{T}_{g}$ is the sub-optimal guard time value. Fig. 5 shows the measured CDF of the relative capacity loss for both guard time selection methods proposed in this section. The results are obtained in the case of CM1 channel realizations. The SNR is fixed to $10 \mathrm{~dB}$. The maximum relative capacity loss value is 0.2 with $T_{\left.g^{(50 \%)}\right)}$ and 0.28 with $T_{g^{(998)}}$. The probability of maximum capacity loss achieved with $T_{g^{(50 \%)}}$ is $10 \%$ and $1 \%$ with $T_{\left.g_{(1998)}\right)}$. The optimal guard time is obtained in $10 \%$ of cases with $T_{g^{(50 \%)}}$ and $1 \%$ of cases with $T_{g_{(99 \%)}}$. In the other channel classes, similar results are obtained.

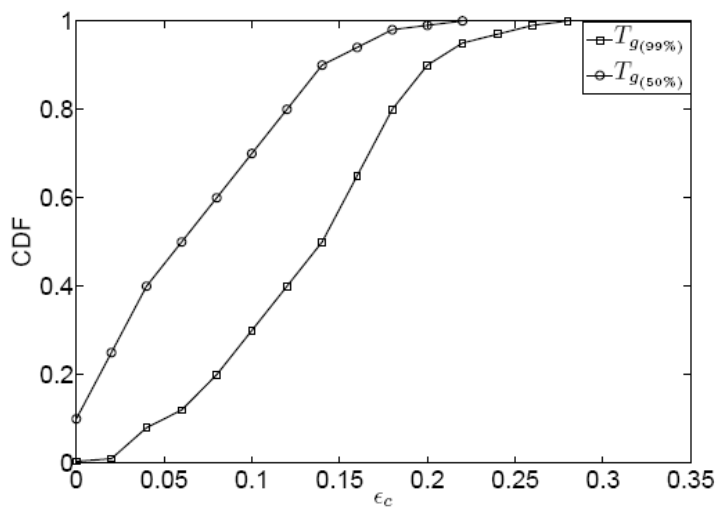

Fig. 5. CDF of relative capacity loss for two guard time values over the CM1 channel model, $\mathrm{SNR}=10 \mathrm{~dB}$.

VI. Alternative Metrics AND Optimization MethoD 
In this section, we describe another methodology to design the guard time that is based on the consideration of alternative metrics. The objective is to define a quantity that is related to the guard time through a parameter value. The proposed metrics are the RMS delay spread, the received signal energy and an approximation of capacity that leads to the energy of the interference. The relation between the optimal parameter $\beta_{\lambda}$, the optimal guard time and the metric L for each channel realization is defined as

$$
\beta=f_{\mathcal{L}}\left(T_{g}^{*}, \lambda\right)
$$

where $\lambda$ is the value of the metric $\mathrm{L}$, and $f_{L}($.$) the function$ that links $\lambda, T_{g}^{*}$ and $\beta$. Now, the method consists in analysing the capacity for a certain channel model. Also, the optimal parameters link the capacity optimal guard time with that obtained using the considered metric are calculated. As a result, the procedure allows avoiding to compute capacity. Firstly, for a certain channel realization the metric $\lambda$ is computed. Then, the guard time is obtained directly by the parameter $\bar{\beta}$ and the value of the metric as

$$
\hat{T}_{g}^{*}=f_{\mathcal{L}}^{-1}(\bar{\beta}, \lambda)
$$

The optimization method used is summarized as follows

- Define the relation between the metric and the channel characteristics

$>$ Capacity calculation using (12)

$>$ Optimal guard time calculation using (13)

$>$ Parameter value calculation using (15)

- Determine one parameter value for all channel realizations

$>$ Guard time calculation using (16)

- Performance evaluation and selection of appropriate metric

$>$ Calculate the capacity attainable with the considered metric

$>$ Calculate the relative capacity loss between the capacity with optimal guard time and the capacity with the sub/optimal guard time.

The procedure above described, allows us to compare the different metrics, extract a sub/optimal guard time value, and compare the attainable capacity with that achievable with the optimal guard time. We now describe in detail the considered metrics.

\section{A. Delay Spread}

The first proposed metric is the RMS delay spread, defined as

$$
\sigma=\sqrt{\frac{\int_{0}^{\infty}(\tau-\mu)^{2} A_{c}(\tau) d \tau}{\int_{0}^{\infty} A_{c}(\tau) d \tau}}
$$

and the average delay spread is

$$
\mu=\frac{\int_{0}^{\infty} \tau A_{c}(\tau) d \tau}{\int_{0}^{\infty} A_{c}(\tau) d \tau}
$$

where $A_{c}(\tau)=|h(\tau)|^{2}$ is the channel power delay profile. The delay spread may change from channel realization to channel realization and depends on the propagation conditions. When $T_{b} \gg \sigma$, the system experiences negligible inter-symbol interference. When $T b$ is within an order of magnitude of $\sigma$, there will be some interference which may or may not degrade the performance. In general, a significant fraction of the received energy is captured within $\beta_{1} \mu$ with $\beta_{1}>0$ [16]. Therefore, in this case the metric $\lambda$ in (15) is the RMS delay spread. The parameter linking the guard time and the RMS delay spread is given as

$$
\beta_{1}=\frac{T_{g}^{*}}{\sigma-T_{p}}
$$

where $T_{g}^{*}$ is defined in (13) and $f_{L}$ in (15) becomes a rational function.

\section{B. Received Signal Energy}

In this section, we define a parameter that links the optimal guard time with the signal energy that can be collected in a certain time window due to the signal spread introduced by the multi-path components. In detail, the parameter $\beta_{2}$ is the fraction of received signal energy that can be collected in a frame of duration $T_{g}^{*}+T_{p}$,

$$
\beta_{2}=\frac{\int_{0}^{T_{g}^{*}+T_{p}}|h(\tau)|^{2} d \tau}{\int_{0}^{T_{c}}|h(\tau)|^{2} d \tau}
$$

where $T_{c}$ is the length of the channel. The parameter value $\beta_{2}$ is takes values in the interval $(0,1]$. The metric $\lambda$ in (15) is the received energy.

In Fig. 6, we show an example of multi-path channel impulse response together with the dotted curve that represents the $\mathrm{CDF}$ of the received signal energy as a function of the frame duration for channel realizations belonging to CM1. Significant signal energy is captured (with high probability) when the frame duration is shorter than the channel maximum duration equal to $30 \mathrm{~ns}$.

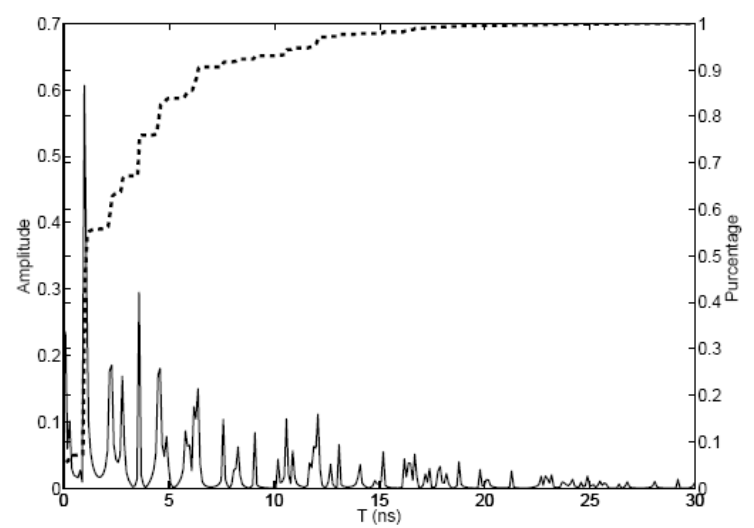

Fig. 6. An example of multi-path channel impulse response and the $\mathrm{CDF}$ of the received signal energy. 


\section{System Capacity Approximation}

The third parameter for the guard time optimization is derived by using a capacity approximation formula. This is obtained when the interference is considered Gaussian as follows

$$
C_{I}=\frac{1}{T_{b} B} \log 2\left(1+\frac{S I N R}{\gamma}\right)
$$

where $\gamma$ is a gap factor that takes into account practical implementation constraints. The sub-optimal guard time is obtained as

$$
T_{g}^{\prime}=\arg \max _{T_{g}}\left(\left(1+\frac{S I N R}{\gamma}\right)^{\frac{1}{T_{b} B}}\right)
$$

A lower bound of (22) is obtained through the Bernoulli inequality

$$
\left(1+\frac{S I N R}{\gamma}\right)^{\frac{1}{T_{b} B}} \geq\left(1+\frac{1}{T_{b} B} \frac{S I N R}{\gamma}\right)
$$

A practical simplified method is to use the lower bound in (23) so that the sub-optimal guard time is

$$
T_{g}^{\prime}=\arg \max _{T_{g}} \frac{S I N R}{\gamma T_{b} B}
$$

The computation of the sub-optimal guard time in (24) has an advantage over (13). Firstly, the computation of the logarithm is avoided. Secondly, (24) requires only the evaluation of the interference power for different values of guard time instead of the computation of the capacity, as in (13).

It follows that the parameter that relates the capacity optimal guard time with the sub-optimal one herein considered is defined as

$$
\beta_{3}=\frac{T_{g}^{*}}{T_{g}^{\prime}}
$$

where $T_{g}^{*}$ is defined by (13).

\section{APPLICATION AND NUMERICAL RESULTS}

Having defined the metrics and the parameters that relate the capacity optimal guard time with that determined with the simplified metric, we now report numerical results assuming again the IEEE802.15.3a channel model presented in Section III. To proceed we first study the $\mathrm{CDF}$ of $\beta_{1}, \beta_{2}$ and $\beta_{3}$ for each channel class. Based on this, we define a single value of the parameter for each channel class and for a certain SNR. Then, the guard time is adapted to a given channel realization by using the considered metric $\lambda$ and the predefined parameter $\beta$.

Fig. 7 shows the measured $\mathrm{CDF}$ of the parameter $\beta_{1}$ according to (19) over CM1 channel realizations. The simulations are realized for three SNR values. These values correspond to a low, a medium and a high SNR. For $\mathrm{SNR}=0 \mathrm{~dB}$, the value of $\beta_{1}$ is in the interval $[0.72$, $0.82]$. These endpoints of the interval decrease to $[0.7,0.8]$ for CM2 channels, to [0.6, 0.73] for CM3 channels and to
$[0.54,0.7]$ for $\mathrm{CM} 4$ channels. For $\mathrm{SNR}=5 \mathrm{~dB}$, the value of $\beta_{1}$ is in the interval $[0.8,0.916]$. These endpoints of the interval decrease to $[0.79,0.9]$ for CM2 channels, to $[0.70$, 0.81] for CM3 channels and to [0.67, 0.78] for CM4 channels. For $\mathrm{SNR}=10 \mathrm{~dB}$, the value of $\beta_{1}$ is in the interval $[0.89,1]$. These endpoints of the interval decrease to $[0.84,0.97]$ for CM2 channels, to [0.76, 0.89] for CM3 channels and to $[0.72,0.86]$ for CM4 channels.

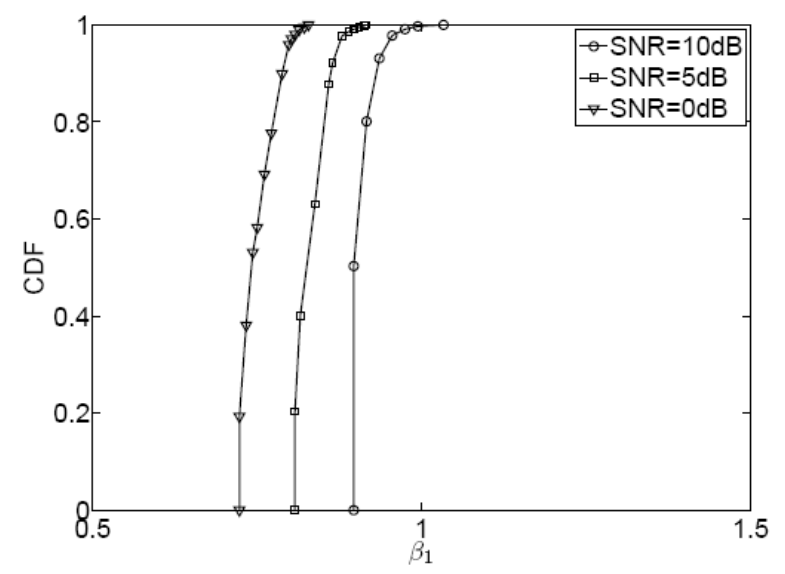

Fig. 7. CDF of parameter $\beta_{1}$ over the $\mathrm{CM} 1$ channel model.

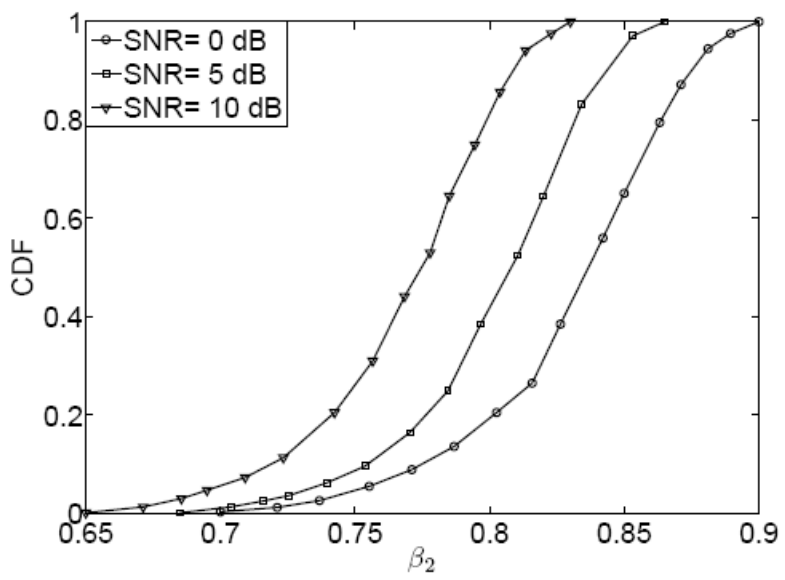

Fig. 8. CDF of parameter $\beta_{2}$ over the CM1 channel model.

Fig. 8 shows the measured $\mathrm{CDF}$ of the parameter $\beta_{2}$ according to (20). The value of $\beta_{2}$ is in the interval [0.65, 0.83 ] in the case of $\mathrm{SNR}=0 \mathrm{~dB}$. For other channel classes, the value of $\beta_{2}$ is in the interval $[0.55,0.78]$ for CM2 channels, in the interval [0.52, 0.77] for CM3 channels and in the interval $[0.45,0.73]$ for CM4 channels. In the case of $\mathrm{SNR}=5 \mathrm{~dB}$, the value of $\beta_{2}$ is in the interval [0.68, $0.86]$. These endpoints of the interval decrease to [0.58, 0.82 ] for $\mathrm{CM} 2$ channels, to [0.54, 0.80] for CM3 channels and to $[0.48,0.76]$ for CM4 channels. In the case of $\mathrm{SNR}=10 \mathrm{~dB}$, the value of $\beta_{2}$ is in the interval [0.7,0.9]. These endpoints of the interval decrease to $[0.6,0.85]$ for CM2 channels, to [0.58, 0.85] for CM3 channels and to $[0.5,0.8]$ for CM4 channels.

Fig. 9 presents the measured CDF of the parameter $\beta_{3}$ according to (24). For $\mathrm{SNR}=0 \mathrm{~dB}$, the value of $\beta_{3}$ is in the interval $[0.45,3]$. The supremum of the interval increases to 3.8 for $\mathrm{CM} 2$ channels, to 5.3 for $\mathrm{CM} 3$ 
channel and to 6.8 for $\mathrm{CM} 4$ channels. For $\mathrm{SNR}=5 \mathrm{~dB}$, the value of $\beta_{3}$ is in the interval $[0.48,3]$. The supremum of the interval increases to 3.9 for CM2 channels, to 5.4 for CM3 channels and to 6.9 for CM4 channels. For $\mathrm{SNR}=10 \mathrm{~dB}$, the value of $\beta_{3}$ is in the interval $[0.53,3]$. The supremum of the interval increases to 4 for CM2 channels, to 5.5 for CM3 channels and to 7 for CM4 channels.

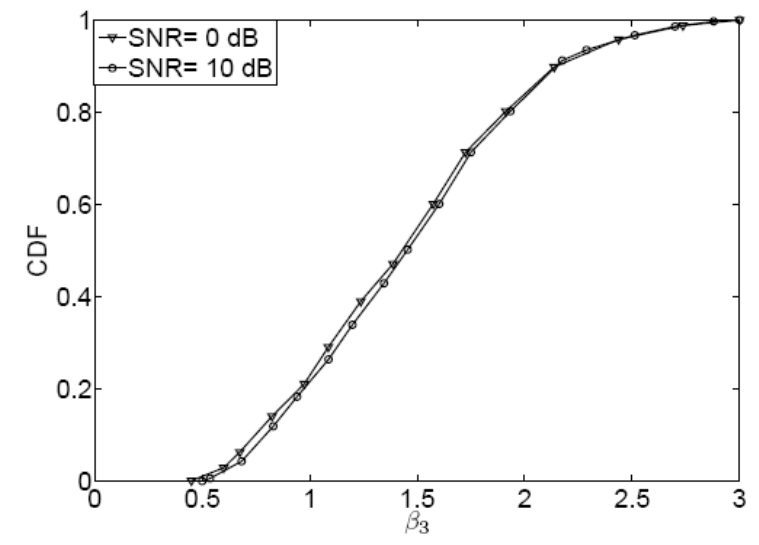

Fig. 9. CDF of parameter $\beta_{3}$ over the CM1 channel model.

Although the parameters depend on the channel class and on the specific impulse response realization, the value of the parameter $\beta_{1}$ is less variable than the parameter value $\beta_{2}$ and $\beta_{3}$ for the same SNR. The value of parameter $\beta_{3}$ is almost identical for different values of SNR.

The best performance is offered by the use of the metric related to the RMS delay spread. However to analyse more deeply the results, the relative capacity loss will be calculated in the next section to validate the proposed approach.

\section{A. Parameter Selection and Performance}

Our strategy is to adapt the guard time length by avoiding the calculation of the capacity formula. To simplify further the problem, we propose to use a single parameter $\beta$ for a certain class and SNR. The parameter is determined by taking the median value of the corresponding CDF. We have found that the resulting system capacity is very close to that attainable with a capacity optimal guard time. We have tabulated the chosen parameter values in Table. III, Table. IV and Table. $\mathrm{V}$ respectively for three SNR levels equal to $=0$ $\mathrm{dB}, 5 \mathrm{~dB}$ and $10 \mathrm{~dB}$.

TABLE III: PARAMETER VALUES, $S N R=0 \mathrm{DB}$

\begin{tabular}{ccccc}
\hline \hline & CM1 & CM2 & CM3 & CM4 \\
\hline$\beta_{1}$ & 0.74 & 0.71 & 0.64 & 0.57 \\
$\beta_{2}$ & 0.77 & 0.64 & 0.62 & 0.52 \\
$\beta_{3}$ & 1.42 & 1.94 & 2.45 & 4.98 \\
\hline \hline
\end{tabular}

TABLE IV: PARAMETER VALUES, $S N R=5 \mathrm{DB}$

\begin{tabular}{ccccc}
\hline \hline & CM1 & CM2 & CM3 & CM4 \\
\hline$\beta_{1}$ & 0.82 & 0.79 & 0.72 & 0.65 \\
$\beta_{2}$ & 0.80 & 0.67 & 0.65 & 0.55 \\
$\beta_{3}$ & 1.44 & 1.95 & 2.46 & 4.99 \\
\hline
\end{tabular}

TABLE V: PARAMETER VALUES, $S N R=10 \mathrm{DB}$

\begin{tabular}{ccccc}
\hline \hline & CM1 & CM2 & CM3 & CM4 \\
\hline$\beta_{1}$ & 0.89 & 0.86 & 0.79 & 0.72 \\
$\beta_{2}$ & 0.836 & 0.70 & 0.68 & 0.58 \\
$\beta_{3}$ & 1.45 & 1.96 & 2.47 & 5 \\
\hline \hline
\end{tabular}

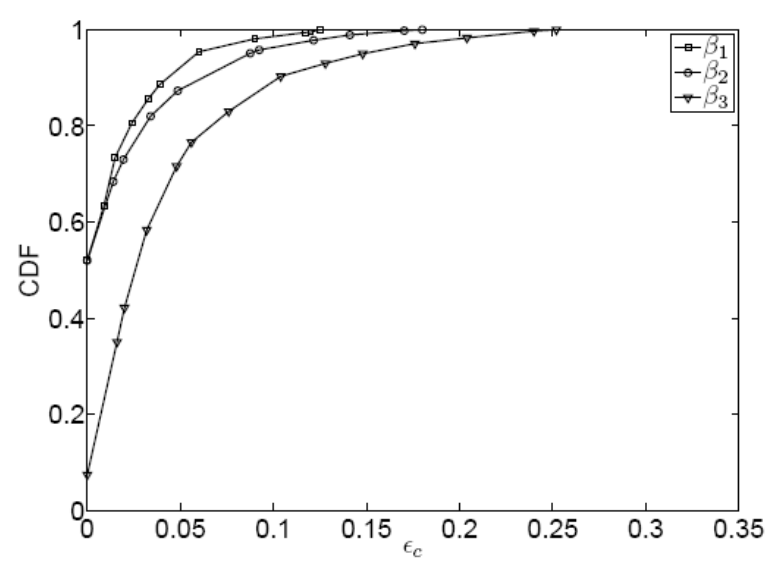

Fig. 10. CDF of relative capacity loss for all three metrics over CM1 channel model. The SNR is fixed to $10 \mathrm{~dB}$.

In order to compare the three metrics, the relative capacity loss is reported in Fig. 10. The results are obtained for $\mathrm{SNR}=10 \mathrm{~dB}$ and the CM1 channel model. Fig. 10 shows the measured CDF of the relative capacity loss when using the three metrics associated to the parameters $\beta$. The delay spread metric $\left(\beta_{1}\right)$ and the signal energy metric $\left(\beta_{2}\right)$ provide less capacity loss than the capacity approximation metric $\left(\beta_{3}\right)$. The maximum relative capacity loss value is 0.12 with $\beta_{1}, 0.16$ with $\beta_{2}$ and 0.25 with $\beta_{3}$. The probability of maximum capacity achieved with $\beta_{1}$ and $\beta_{3}$ is $52 \%$ and $9 \%$ for $\beta_{3}$. The optimal guard time is obtained in $52 \%$ of cases with $\beta_{1}$ and $\beta_{2}$. Although not shown, with the other channel models, the maximum relative capacity loss value with $\beta_{1}$ is 0.13 in the CM2 channels, 0.14 in CM3 channels and 0.15 in CM4 channels. For $\beta_{2}$, the maximum relative capacity loss value is 0.16 in CM2 channels, 0.18 in CM3 channels and 0.19 with the CM4 channel model. For $\beta_{3}$, the maximum relative capacity loss value is 0.25 in CM2 channels, 0.26 in CM3 channels and 0.28 in CM4 channels.

Although, all three simplified metrics provide small relative capacity losses, the best approach is to use the delay spread metric with the adjustment parameter $\beta_{1}$.

Although not shown similar conclusions are reached for other SNR values. Finally, it should be noted that the use of the delay spread metric is significantly less complex than determining the capacity optimal guard time described in Section IV.

\section{CONCLUSION}

In this paper, we have examined the problem of designing the guard time in BPSK UWB communications. We have shown that the use of a guard time adjusted to the current channel conditions is beneficial in terms of 
maximizing the system capacity. Ideally, this should be done by adjusting the guard time to the specific channel impulse response so that the capacity is maximized. However, this requires an exhaustive guard time search which is a computationally intense task. Therefore, we have considered the use of a constant guard time value for all channel realizations belonging to a certain channel class. Another approach is to adapt the guard time to the channel realization but such an adaptation is done by resorting on a simplified metric, namely the delay spread, the signal energy, or a lower bound of the capacity. The resulting guard time is then adjusted by a factor that depends on the channel class and operating SNR. Numerical results for typical indoor UWB channels have shown that a significant gain can be achieved w.r.t. the use of a conservative guard time length equal to the maximum channel duration. In particular, the metric based on the delay spread, appropriately adjusted, provides the smallest loss compared to the capacity optimal delay spread.

\section{APPENDIX A: CAPACITY CALCULATION}

The mutual information $\mathrm{I}(\mathrm{X}, \mathrm{Y})$ is a function of the signal power $E_{s}$ and the noise power $N_{0}$. The capacity for BPSK depends on these parameters only through their ratio, the SNR $E / N_{0}$. To show this, we replace $Y$ by $Y / N_{0}$ to get the model

$$
Y=\sqrt{S N R} X+N, N \sim \mathcal{N}\left(0, N_{0}\right)
$$

For notational simplicity, set $A=\sqrt{S N R}$ We have

$$
\begin{aligned}
& p(Y \mid+1)=\frac{1}{\sqrt{2 \pi}} \exp \left(-(Y-A)^{2} / 2\right) \\
& p(Y \mid-1)=\frac{1}{\sqrt{2 \pi}} \exp \left(-(Y+A)^{2} / 2\right)
\end{aligned}
$$

and

$$
\begin{gathered}
p(Y)=\frac{1}{2} p(Y \mid+1)+\frac{1}{2} p(Y \mid-1) \\
p(Y)=\frac{1}{2} \frac{1}{\sqrt{2 \pi}} \sum_{s=1}^{2} \exp \left(-\left(Y+b_{s} A\right)^{2} / 2\right)
\end{gathered}
$$

where $b_{s}=\{-1,1\}$

We can now compute

$$
I(X, Y)=h(Y)-h(Y, X)
$$

As in [15], we can show that $h(Y \mid X)=h(Z)=$ $1 / 2 \log _{2}\left(2 \pi e N_{0}\right)$ We can now compute

$$
h(Y)=-\int \log _{2}(p(Y)) p(Y)
$$

by numerical integration, plugging in (28). An alternative approach, which is particularly useful for more complicated constellations and channel models, is to use Monte Carlo integration (i.e., simulation-based empirical averaging) for computing the expectation $h(Y)=-E\left[\log _{2}\right.$ $p(Y)]$. For this method, we generate i.i.d. samples $Y_{i}$ using the model (26), and then use the estimate

$$
h=-\frac{1}{n} \sum_{1}^{n} \log _{2} p\left(Y_{i}\right)
$$

then the capacity

$$
\begin{gathered}
C=-E\left[\log _{2} p(Y)\right]-1 / 2 \log _{2}\left(2 \pi e N_{0}\right) \\
C=-E\left[\log _{2} \frac{1}{2} \frac{1}{\sqrt{2 \pi}} \sum_{i=1}^{2} \exp \left(-\left(Y+b_{i} A\right)^{2} / 2\right)\right] \\
-1 / 2 \log _{2}\left(2 \pi e N_{0}\right)
\end{gathered}
$$

\section{Capacity with interference}

1) One bit interference.

With only one interferer, we obtain

$$
\begin{aligned}
p(Y) & =\frac{1}{4} p\left(Y \mid\left(+1, a_{1}\right)\right)+\frac{1}{4} p\left(Y \mid\left(+1,-a_{1}\right)\right) \\
& +\frac{1}{4} p\left(Y \mid\left(-1,-a_{1}\right)\right)+\frac{1}{4} p\left(Y \mid\left(-1, a_{1}\right)\right)
\end{aligned}
$$

where $a_{1}$ is the interferer amplitude

$$
\begin{aligned}
p(Y)= & \frac{1}{4} \frac{1}{\sqrt{2 \pi}}\left(\exp -\frac{\left(Y-A+a_{1}\right)^{2}}{2}\right. \\
& +\exp -\frac{\left(Y-A-a_{1}\right)^{2}}{2} \\
& +\exp -\frac{\left(Y+A+a_{1}\right)^{2}}{2} \\
& \left.+\exp -\frac{\left(Y+A-a_{1}\right)^{2}}{2}\right)
\end{aligned}
$$

then

$$
\begin{aligned}
h(Y)= & -E\left[\operatorname { l o g } _ { 2 } \left(\frac { 1 } { 4 } \frac { 1 } { \sqrt { 2 \pi } } \left(\exp -\frac{\left(Y-A+a_{1}\right)^{2}}{2}\right.\right.\right. \\
& +\exp -\frac{\left(Y-A-a_{1}\right)^{2}}{2} \\
& +\exp -\frac{\left(Y+A+a_{1}\right)^{2}}{2} \\
& \left.\left.\left.+\exp -\frac{\left(Y+A-a_{1}\right)^{2}}{2}\right)\right)\right]
\end{aligned}
$$

2) Two bits interference.

With two interferers, we obtain

$$
\begin{gathered}
p(Y)=\frac{1}{8} p\left(Y \mid\left(+1, a_{1}, a_{2}\right)\right)+\frac{1}{8} p\left(Y \mid\left(+1, a_{1},-a_{2}\right)\right) \\
+\frac{1}{8} p\left(Y \mid\left(+1,-a_{1},-a_{2}\right)\right)+\frac{1}{8} p\left(Y \mid\left(+1,-a_{1}, a_{2}\right)\right) \\
+\frac{1}{8} p\left(Y \mid\left(-1, a_{1}, a_{2}\right)\right)+\frac{1}{8} p\left(Y \mid\left(-1, a_{1},-a_{2}\right)\right) \\
+\frac{1}{8} p\left(Y \mid\left(-1,-a_{1},-a_{2}\right)\right)+\frac{1}{8} p\left(Y \mid\left(-1,-a_{1}, a_{2}\right)\right) \\
p(Y)=\frac{1}{8} \frac{1}{\sqrt{2 \pi}}\left(\exp -\frac{\left(Y-A+a_{1}+a_{2}\right)^{2}}{2}\right. \\
+\exp -\frac{\left(Y-A+a_{1}-a_{2}\right)^{2}}{2} \\
+\exp -\frac{\left(Y-A-a_{1}-a_{2}\right)^{2}}{2}
\end{gathered}
$$




$$
\begin{aligned}
& +\exp -\frac{\left(Y-A-a_{1}+a_{2}\right)^{2}}{2} \\
& +\exp -\frac{\left(Y+A+a_{1}+a_{2}\right)^{2}}{2} \\
& +\exp -\frac{\left(Y+A+a_{1}-a_{2}\right)^{2}}{2} \\
& +\exp -\frac{\left(Y+A-a_{1}+a_{2}\right)^{2}}{2} \\
& \left.+\exp -\frac{\left(Y+A-a_{1}-a_{2}\right)^{2}}{2}\right)
\end{aligned}
$$

then

$$
\begin{aligned}
h(Y)= & -E\left[\operatorname { l o g } _ { 2 } \left(\frac { 1 } { 8 } \frac { 1 } { \sqrt { 2 \pi } } \left(\exp -\frac{\left(Y-A+a_{1}+a_{2}\right)^{2}}{2}\right.\right.\right. \\
& +\exp -\frac{\left(Y-A+a_{1}-a_{2}\right)^{2}}{2} \\
& +\exp -\frac{\left(Y-A-a_{1}-a_{2}\right)^{2}}{2} \\
& +\exp -\frac{\left(Y-A-a_{1}+a_{2}\right)^{2}}{2} \\
& +\exp -\frac{\left(Y+A+a_{1}+a_{2}\right)^{2}}{2} \\
& +\exp -\frac{\left(Y+A+a_{1}-a_{2}\right)^{2}}{2} \\
& +\exp -\frac{\left(Y+A-a_{1}+a_{2}\right)^{2}}{2} \\
& \left.\left.\left.+\exp -\frac{\left(Y+A-a_{1}-a_{2}\right)^{2}}{2}\right)\right)\right]
\end{aligned}
$$

3) $n$ bits interference.

More in general, with $n$ binary interferers, we have that

$$
\begin{aligned}
& h(Y)=-E\left[\operatorname { l o g } _ { 2 } \left(\frac{1}{2^{n+1}} \frac{1}{\sqrt{2 \pi}}\right.\right. \\
& \left.\left.\sum_{s=1}^{2} \sum_{i=1}^{2^{n}} \exp \left(-\frac{\left(y-b_{s} A+\sum_{j=1}^{n} a_{j} \alpha_{j, i}\right)^{2}}{2}\right)\right)\right]
\end{aligned}
$$

It follows that the capacity with BPSK and $n$ binary interferers can be obtained as

$$
\begin{aligned}
C & =-E\left[\operatorname { l o g } _ { 2 } \left(\frac{1}{2^{n+1}} \frac{1}{\sqrt{2 \pi}}\right.\right. \\
& \left.\left.\sum_{s=1}^{2} \sum_{i=1}^{2^{n}} \exp \left(-\frac{\left(y-b_{s} A+\sum_{j=1}^{n} a_{j} \alpha_{j, i}\right)^{2}}{2}\right)\right)\right] \\
& -1 / 2 \log _{2}\left(2 \pi e N_{0}\right)
\end{aligned}
$$

where $n$ is the number of interferers, $a_{j}$ is the amplitude of the $j-t h$ interfering bit, $\alpha_{j, i}$ is a binary value corresponding to the possible combinations of interferers with dimension $\{n, 2 n\}$ and $b_{s}=\{-1,1\}$. As an example for $n=2$ :

$$
\alpha_{j, i}=\left(\begin{array}{cc}
+1 & -1 \\
+1 & +1 \\
-1 & +1 \\
-1 & -1
\end{array}\right)
$$

\section{REFERENCES}

[1] I. Oppermann, M. Hamalainen, and J. Iinatti, Eds., UWB Theory and Applications. John Wiley \& Sons Ltd, 2004.

[2] S. G. Glisic, Advanced Wireless Networks 4G Technologies, John Wiley \& Sons Ltd, 2006.

[3] H. Nikookar and R. Prasad, Introduction to Ultra Wideand for Wireless Communciations, Springer, 2009.

[4] M. Ghavami, L. B. Michael, and R. Kohno, Ultra Wide Band: Signal and Systems in Communication Engineering, S. Hinton, Ed. Willey, 2007.

[5] M. Win and R. Scholtz, "Impulse radio: How it works," Communications Letters, IEEE, vol. 2, no. 2, pp. 36-38, Feb 1998.

[6] L. Yang and G. Giannakis, "Ultra-wideband communications: An idea whose time has come," Signal Processing Magazine, IEEE, vol. 21 , no. 6, pp. 26-54, Nov 2004

[7] K. Witrisal, "Noncoherent autocorrelation detection of orthogonal multicarrier UWB signals," in International Conference on UltraWideband, IEEE, vol. 2, Sept 2008, pp. $161-164$.

[8] MAC-PHY Interface Specification, WiMedia Alliance MACPHY Working Group Std., 2009.

[9] Z. Zhao-yang and L. Li-feng, "A novel OFDM transmission scheme with length-adaptive cyclic prefix," Journal of Zhejiang University Science, vol. 2004, pp. 1336-1342, 2004.

[10] A. Tonello, S. D’Alessandro, and L. Lampe, "Cyclic prefix design and allocation in bit-loaded OFDM over power line communication channels," IEEE Transactions on Communications, vol. 58, no. 11, pp. 3265 -3276, Nov 2010.

[11] J. Foerster, "Channel modeling sub-comittee report final," IEEE P802.15 Working Group for Wireless Personal Area Networks (WPANs), Tech. Rep., Feb. 2003.

[12] A. M. Tonello and R. Rinaldo, "Frequency domain channel estimation and detection for impulse radio systems," in WPMC, Italy, Sep 2004.

[13] J. Proakis, Digital Communication, Wiley, 2002.

[14] A. Saleh and R. Valenzuela, "A statistical model for indoor multipath propagation," IEEE Journal on Selected Areas in Communications, vol. 5, no. 2, pp. 128-137, Feb 1987.

[15] U. Madhow, Fundamental of Digital Communication, Cambridge University Pess, 2008.

[16] A. Goldsmith, Wireless Communication, Stanford University, 2005.

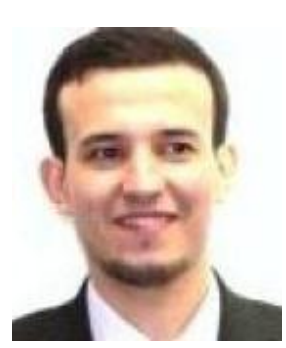

Abdallah Hamini received M.Sc. degree in signal, telecommunications, image, networks and multimedia from the Institute of Galilée in Paris, France, in 2009. In March 2013, he received $\mathrm{PhD}$ degree in electronics and telecommunication, from both the National Institute of Applied Sciences of Rennes (INSA), France and the University of Udine, Italy. His main research interests is on the area of signal processing and digital 


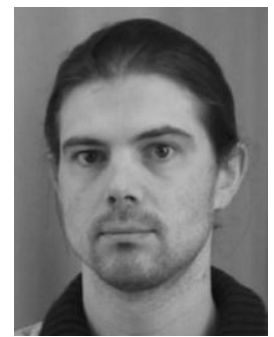

Jean-Yves Baudais received the M.Sc. degree, and $\mathrm{PhD}$ degree in electrical engineering from the National Institute of Applied Sciences of Rennes (INSA), France, in 1997 and 2001 respectively. In 2002, he joined the French National Centre for Scientific Research (CNRS), where he is now researcher in the Institute for Electronics and Telecommunications of Rennes (IETR), Digital Communication Systems team (SCN). His general interests lie in the areas of signal processing and digital communications. Current research focuses on transmitter design and receiver diversity techniques for multiuser and multicarrier communication including space-time coding. He is currently the head of SCN team.

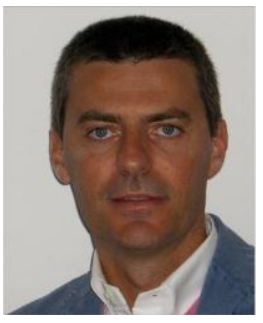

Andrea M. Tonello is an Aggregate Professor at the University of Udine, Italy (since 2003) where he leads the Wireless and Power Line Communication Lab. He is also the founder and president of WiTiKee, a university spinoff company working in the field of telecommunications for the smart grid. From 1997 to 2002 he has been with Bell Labs Lucent Technologies firstly as a Member of Technical Staff and then as a Technical Manager at the Advanced Wireless Technology Laboratory, Whippany, NJ and the Managing Director of the Bell Labs Italy division. He obtained the Laurea degree (1996, summa cum laude) and the Doctor of Research degree in electronics and telecommunications (2003) from the University of Padova, Italy. In 2013, Dr. Tonello received the Italian Full Professor Habilitation. He was awarded several other recognition among which the Lucent Bell Labs Recognition of Excellence award
(1999) , the Distinguished Visiting Fellowship from the Royal Academy of Engineering, UK (2010) and the Distinguished Lecturer Award by the IEEE Vehicular Technology Society (2011-13 and 2013-15). He also received (as co-author) five best paper awards. He is the Vice-chair of the IEEE Communications Society Technical Committee on Power Line Communications. He serves/ed as an Associate Editor for the IEEE Transactions on Vehicular Technology (2007-2013), for the IEEE Transactions on Communications (since 2012) and IEEE Access (since 2013). He served as the general chair of IEEE ISPLC 2011 and he is the general co-chair of IEEE Smart Grid Comm 2014.

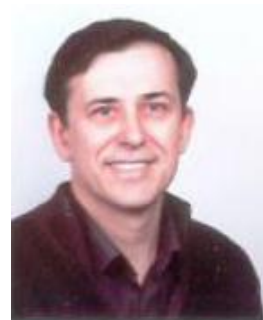

Jean-François Hélard received his Dipl.- Ing. and his Ph.D in electronics and signal processing from the National Institute of Applied Sciences (INSA) in Rennes, France, in 1981 and 1992, respectively. From 1982 to 1997, he was research engineer and then head of channel coding for the digital broadcasting research group at France Telecom Research Center in Rennes. In 1997, he joined INSA, where he is currently Professor and Director of Research of the Institute. $\mathrm{He}$ is also Deputy Director of the Rennes Institute for Electronics and Telecommunications (IETR), created in 2002 in association with the CNRS. His research interests lie in signal processing techniques for digital communications, such as space-time and channel coding, multicarrier modulation, as well as spread-spectrum and multi-user communications. He is involved in several European and national research projects in the fields of digital video terrestrial broadcasting, mobile radio communications and cellular networks, power-line and ultra-wide-band communications, cooperative communications and relaying techniques. Prof. JF. Hélard is a senior member of IEEE, author and co-author of more than 200 technical papers in international scientific journals and conferences, and holds 14 European patents. 